\title{
Three Logico-Ontological Notions and Mereology
}

Uwe Meixner

\section{Axiomatic Method}

The axiomatic method is realized, also in the mereological case, by specifying an axiomatic system $\mathrm{S}$, consisting of the axioms of $S$ and the logic of $S$. The axioms of $S$ are certain basic statements, and the logic of $\mathrm{S}$ is a set of basic inference-rules which can be used to generate further statements from given statements (ultimately from the axioms). The specification of $\mathrm{S}$ must be effective, that is, it must be in every case decidable whether or not a given statement belongs to the axioms of $\mathrm{S}$, and whether or not a given inference-rule belongs to the logic of $\mathrm{S}$.

Relative to the axiomatic system $\mathrm{S}$ - the axioms plus the logic - a notion of provability is recursively defined: (1) the axioms of $\mathrm{S}$ are provable in S; (2) if the premise(s) of an inference-rule of the logic of S are provable in $\mathrm{S}$, then also the conclusion of that inference-rule is provable in S; (3) Only statements that can be obtained by (1) and (2) are provable in $\mathrm{S}$. Those statements that are provable in $\mathrm{S}$ and that are not axioms of $\mathrm{S}$ are the theorems of $S$.

Normally, the syntax of a language used in an axiomatic system allows only such well-formed statements as are finite-length strings of discrete signs; and normally, the inference-rules of an axiomatic system have only finitely many premises. Normally, the inference-rules of an axiomatic system are validity-preserving, that is, if their premises are valid, then their conclusion must be valid, too; and normally, the axioms of an axiomatic system are valid statements. Normally, validity entails truth. Normally, the axioms and basic inference-rules of an axiomatic system are easily describable (though it may consist of infinitely many axioms and basic inference-rules), and their description will fit on a few printed pages, perhaps on only one page, perhaps on just a few lines. If an axiomatic system is normal in the sense of displaying all six of the aspects of normality just specified, then that system fulfils the purpose of describing validly (hence truthfully), rigorously, and compendiously a certain region of being.

A normal axiomatic system $\mathrm{S}$ is complete with regard to validity in the language of $\mathrm{S}$ if all the valid statements in that language are provable in 
S. A normal axiomatic system $\mathrm{S}$ is minimal if it has no proper part $\mathrm{S}^{\prime}$ which is such that the very same statements are provable in $\mathrm{S}^{\prime}$ that are provable in S. Completeness and minimality are more or less aesthetic virtues in a normal axiomatic system (and completeness, in fact, a virtue that is realizable only within very narrow confines).

Regarding the interpretation of a normal axiomatic system $\mathrm{S}$, there is a fundamental alternative: either the language of $\mathrm{S}$ is completely interpreted, or it is not. The interesting case is the latter. In this latter case, S may be taken to enumerate valid (hence true) statements; but it is not entirely determined about what they are valid statements. The only thing (provisionally) determined is that the statements of $\mathrm{S}$ (its axioms and theorems) are to be understood in such a way as to be valid (hence true); but usually there are various ways known of understanding them thus. Moreover, if the language of $\mathrm{S}$ is not completely interpreted, then alternative (though in certain respects similar) interpretations of it may suggest themselves, and accordingly also a multitude of axiomatic systems that are alternatives to $\mathrm{S}$ - even systems $\mathrm{S}^{\prime}$ that allow the proof of statements of which the negations are provable in S. Nevertheless, both $\mathrm{S}$ and $\mathrm{S}^{\prime}$ can each be a normal axiomatic system. Therefore, $\mathrm{S}$ can be taken to enumerate valid statements, and $\mathrm{S}^{\prime}$ can also be taken to enumerate valid statements. This is made possible by the fact that the language of $\mathrm{S}$ - which is also the language of $\mathrm{S}^{\prime}$ - is incompletely interpreted: that language - in its given state of interpretation - allows that all the statements of $\mathrm{S}$ be valid (which means: there is a completion of the language's incomplete interpretation according to which all the statements of $S$ are valid), but also allows that all the statements of $S^{\prime}$ be valid (which means: there is another completion of that interpretation according to which all the statements of $\mathrm{S}^{\prime}$ are valid).

For illustration, consider the mereological case. We have a language $\mathrm{L}$ of first-order predicate logic with identity, and in that language a special predicate, $\mathrm{P}(\mathrm{x}, \mathrm{y})$, to be read as: " $\mathrm{x}$ is a part of $\mathrm{y}$ ". An axiomatic mereological system with respect to $\mathrm{L}$ is an axiomatic system, formulated in $\mathrm{L}$, in whose axioms the predicate $\mathrm{P}(\mathrm{x}, \mathrm{y})$ is the most prominent predicate. Consider the following three axiomatic mereological systems with respect to $\mathrm{L}$, of which in each case only the first three axioms are stated: 
MS1

The logic of MS1: first-order predicate logic with identity.

The axioms of MS1:

$\forall \mathrm{x} \forall \mathrm{y} \forall \mathrm{z}(\mathrm{P}(\mathrm{x}, \mathrm{y}) \wedge \mathrm{P}(\mathrm{y}, \mathrm{z}) \supset \mathrm{P}(\mathrm{x}, \mathrm{z}))$,

$\forall \mathrm{xP}(\mathrm{x}, \mathrm{x})$

$\forall \mathrm{x} \forall \mathrm{y}(\mathrm{P}(\mathrm{x}, \mathrm{y}) \wedge \mathrm{P}(\mathrm{y}, \mathrm{x}) \supset \mathrm{x}=\mathrm{y})$, etc.

MS2

The logic of MS2: the logic of MS1.

The axioms of MS2:

$\forall \mathrm{x} \forall \mathrm{y} \forall \mathrm{z}(\mathrm{P}(\mathrm{x}, \mathrm{y}) \wedge \mathrm{P}(\mathrm{y}, \mathrm{z}) \supset \mathrm{P}(\mathrm{x}, \mathrm{z}))$,

$\forall \mathrm{x} \forall \mathrm{y}[\mathrm{P}(\mathrm{x}, \mathrm{y}) \supset \exists \mathrm{z}(\mathrm{P}(\mathrm{z}, \mathrm{y}) \wedge \mathrm{z} \neq \mathrm{x} \wedge \neg \exists \mathrm{u}(\mathrm{P}(\mathrm{u}, \mathrm{x}) \wedge \mathrm{P}(\mathrm{u}, \mathrm{z})))]$,

$\forall \mathrm{y} \exists \mathrm{xP}(\mathrm{x}, \mathrm{y})$, etc.

\section{MS3}

The logic of MS3: the logic of MS2.

The axioms of MS3:

$\forall \mathrm{x} \forall \mathrm{y} \forall \mathrm{z}(\mathrm{P}(\mathrm{x}, \mathrm{y}) \wedge \mathrm{P}(\mathrm{y}, \mathrm{z}) \supset \mathrm{P}(\mathrm{x}, \mathrm{z}))$,

$\forall \mathrm{x} \forall \mathrm{y}(\mathrm{P}(\mathrm{x}, \mathrm{y}) \supset \neg \mathrm{P}(\mathrm{y}, \mathrm{x}))$,

$\exists y \forall x \neg P(x, y)$, etc.

These three systems are pairwise contradictory to each other, in the sense that, for each pair, a statement is provable in one member of the pair of which the negation is provable in the other. Yet, each can be a normal axiomatic mereological system with respect to L, depending on how the interpretation of $\mathrm{L}$ is completed. If the universe of discourse of L comprises precisely the subsets of the set of human beings, and $\mathrm{P}(\mathrm{x}, \mathrm{y})$ means as much as " $x$ is a proper or improper subset of $y$," then the three stated axioms of MS1 are valid. If, however, the universe of discourse of $\mathrm{L}$ comprises precisely the volumes of space, and $\mathrm{P}(\mathrm{x}, \mathrm{y})$ means as much as " $x$ is a proper sub-volume of $y$," then the three stated axioms of MS2 are valid. If, finally, the universe of discourse of L comprises precisely the natural numbers and $\mathrm{P}(\mathrm{x}, \mathrm{y})$ means as much as " $\mathrm{x}$ is a proper subnumber of $y$," then the three stated axioms of MS3 are valid. 


\section{Abstract(a)}

All abstract individuals have neither spatial nor temporal parts, or in other words: they have neither a spatial nor a temporal localization in a literal - non-analogical — sense. Perhaps all individuals that have neither spatial nor temporal parts are abstract, perhaps not: God, angels, and souls would be individuals that have neither spatial nor temporal parts, yet one would not call any one of them "abstract".

Not only certain individuals but also certain non-individuals are abstract. In fact, there is a longstanding tendency in ontology to consider all non-individuals to be abstract entities, the rationale for this being that all non-individuals have neither spatial nor temporal parts (in a literal sense). But, as in the case of individuals, so also in the case of nonindividuals (for example, universals and states of affairs): the absence of spatial and temporal parts does not appear to be a sufficient condition for abstractness (though it is a necessary condition in both cases). It just does not seem appropriate to call, say, the state of affairs of the earth's revolving around the sun an abstract entity.

It has been suggested that what makes an entity abstract is its lack of causal powers. But the absence of causal powers, too, is no sufficient condition for abstractness (although it is a necessary condition for it), because not all causal epiphenomena are bound to be abstract. If some conscious experiences had no causal powers, it would certainly not make them abstract. Nor would $I$ be abstract if I - in contrast to my brain had no causal powers.

Whatever may be the precise meaning of abstractness, propositions and concepts belong to ontological categories that, with great plausibility, are abstract throughout: very plausibly, all propositions and all concepts are abstract. This implies that propositions and concepts have neither spatial nor temporal parts. Their lack of spatial and temporal parts, however, does not prevent propositions and concepts from having, and being, parts in some sense, and hence it does not prevent them from having their own abstract mereologies.

The mereology of propositions can be built on the notion of logical part, where proposition $p$ is a logical part of proposition $q$ if, and only if, $q$ logically entails $p$. The intended logical entailment is logical entailment broadly conceived; it is not logical entailment as codified in some logical system, say, first-order predicate logic (but logical entailment 
broadly conceived does of course extensionally comprise the entailment relation of first-order predicate logic: every instance of the latter relation is also an instance of the former).

The mereology of concepts can be built on top of the mereology of propositions by making use of the following definition, which extends the notion of logical part from propositions to concepts:

The concept $F$ is a logical part of the concept $G$ if, and only if, (1) F and $\mathrm{G}$ are meaningful for exactly the same entities, and (2), for all $\mathrm{x}$ for which $\mathrm{F}$ is meaningful, the proposition that $\mathrm{F}$ applies to $\mathrm{x}$ is a logical part of the proposition that $\mathrm{G}$ applies to $\mathrm{x}$.

Thus, for example, the concept of extendedness is a logical part of the concept of colouredness, because (1) both concepts are meaningful for exactly the same entities and because (2) for all $x$ for which extendedness is meaningful: the proposition that extendedness applies to $\mathrm{x}$ is a logical part of the proposition that colouredness applies to $\mathrm{x}$.

A mereology with primitive part-relation (and not with some other mereological relation as primitive, as for example the relation of overlap) is either a proper-parts or a proper-or-improper-parts mereology, depending on how the basic part-relation is conceived of. Clearly, the mereology of propositions, as based on the notion of logical part, understood in the sense specified above, is a proper-or-improper-parts mereology. For the part-relation of that mereology, transitivity and reflexivity hold (for all entities in its field, that is: for all propositions) - this is no news compared to other proper-or-improper-parts mereologies. But an abstract mereology — for example, the mereology of propositions differs significantly from a usual proper-or-improper-parts mereology. In contrast to a usual mereology of this kind, the principle of mereological extensionality - according to which entities that are parts of each other are identical to each other - fails to hold in the case of the mereology of propositions: Even though the proposition that Jack is older than Mack and the proposition that Mack is younger than Jack are logical parts of each other, the two propositions are two propositions, and not one and the same. Moreover, it is not a usual feature of mereologies that, according to them, some entity that belongs to the field of their part-relation is a part of every entity that belongs to that field. For example, there certainly is no volume of space which is a sub-volume of every volume of space. In contrast, there are many - indeed, infinitely 
many - propositions which are a logical part of every proposition: the proposition that $1=1$, the proposition that what is not extended is not coloured, the proposition that not every proposition is false, etc.

Especially the feature of the mereology of propositions that has just been described may suggest to some that the so-called mereology of propositions is not really — but only analogically — a mereology. Yet, one may well ask: what is an intuitively satisfactory criterion according to which one is to decide whether a theory is really (literally, genuinely) a mereology or not? Here is such a criterion:

A mereology-like theory is a genuine mereology if, and only if, its part-relation satisfies the following schema: For all $\mathrm{x}$ and $\mathrm{y}$ : $\mathrm{x}$ is a part of $\mathrm{y}$ only if the conjunction (or sum) of $\mathrm{x}$ and $\mathrm{y}$ is identical to $\mathrm{y}$.

And, as a matter of fact, we find that the part-relation of the mereology of propositions does not appear to satisfy that schema: The proposition that Mack is male is a logical part of the proposition that Mack is the son of Jack. But it does not seem to be the case that the conjunction of the two propositions is identical to the proposition that Mack is the son of Jack: the proposition that Mack is male and the son of Jack seems to be obviously different from the proposition that Mack is the son of Jack. Hence one is quite justified in concluding that the mereology of propositions is not a genuine mereology, but a mereology only in an analogical sense. However, the situation changes fundamentally if propositions - quite disregarding the plausibility of their uniform abstractness - are identified with coarse-grained states of affairs, which to some thinkers has not seemed a totally inadequate thing to do.

\section{Universals}

Universals belong to those entities that have neither spatial nor temporal parts, and therefore have neither a (literal) spatial nor a (literal) temporal localization. Universals are either non-predicative or predicative. The non-predicative universals are also called types or type-objects (for example, the letter A). The predicative universals, in turn, are divided into the properties and the relations. Types are closely related to properties: there is a property $\mathrm{p}(\mathrm{T})$ corresponding one-to-one to each type $\mathrm{T}$, such that $\mathrm{x}$ exemplifies/instantiates $\mathrm{T}$ if, and only if, $\mathrm{x}$ exemplifies/instantiates $\mathrm{p}(\mathrm{T})$. 
Predicative universals should be distinguished from concepts, just as states of affairs should be distinguished from propositions. But just as there is a certain analogy between states of affairs and propositions, so there is also a certain analogy between predicative universals and concept. In particular, there is an analogy between properties and monadic concepts, and an analogy between relations and polyadic concepts. The analogy is of such a strong kind that names for properties can also be used as names for monadic concepts, and names for relations also as names for polyadic concepts. Thus, "love" can both function as a name for a certain dyadic relation, and as a name for a certain dyadic concept. In those cases where the context does not already make it clear what is being referred to, the name can easily be disambiguated: "the relation of love," "the concept of love." The situation is entirely the same in the case of states of affairs and propositions: "that the moon revolves around the earth" can function both as a name for a state of affairs, and as a name for a proposition; putting "the state of affairs" or "the proposition" to the left of the "that"-phrase will make it clear, if need be, what is being referred to.

Moreover, predicative universals and states of affairs belong together in a way that is analogous to the way in which concepts and propositions belong together. What is that way? Concepts are prominent constituents in the composition of propositions. Analogously, a predicative universal $\mathrm{U}$ together with the right number $\mathrm{N}$ of ordered entities $\mathrm{X}_{1}, \ldots, \mathrm{X}_{\mathrm{N}}$, each of which is - in its place — of the right kind, constitute a state of affairs: the state of affairs which is the composition of $U$ with $X_{1}, \ldots$, $\mathrm{X}_{\mathrm{N}}$, in short: $\left[\mathrm{U}, \mathrm{X}_{1}, \ldots, \mathrm{X}_{\mathrm{N}}\right]$. The just-mentioned rightness for composition is dictated by the so-called type of $\mathrm{U}$, by its composition-profile, so to speak; if that composition-profile is not respected, the compositionresult will not be a state of affairs. For example, [Younger, Mack, Jack] - the composition of the dyadic relation Younger with, first, the human individual Mack, and, second, the human individual Jack - is a state of affairs because it respects the type of Younger; it is the state of affairs that Mack is younger than Jack. But neither [Younger, Mack] nor [Younger, Mack, Younger] are states of affairs, because they do not respect the type of Younger.

A predicative universal $\mathrm{U}$ is exemplified by the entities $\mathrm{X}_{1}, \ldots, \mathrm{X}_{\mathrm{N}}$ (in the given order) if, and only if, $\left[\mathrm{U}, \mathrm{X}_{1}, \ldots, \mathrm{X}_{\mathrm{N}}\right]$ is an obtaining state of 
affairs (a fact). Sometimes instantiation is distinguished from exemplification, such that an apple, for example, is taken to exemplify, but not to instantiate, the property of being red, whereas a red-trope, found on that apple, is indeed taken to instantiate that property. It seems best to treat instantiation as a species of exemplification: instantiation is exemplification by individuals which are not substances (that is, by entities that are so-called individual accidents).

Though universals have neither spatial nor temporal parts, this does not prevent them from having, and being, parts in some sense. In a sense, a universal is a part of all the state of affairs it helps to compose. But since universals and states of affairs differ in ontological category, it seems rather more appropriate to say that a universal is a constituent of all the states of affairs it helps to compose than that it is a part of them. Part-relations between homocategorial entities differ vastly from partrelations between heterocategorial entities, and there seems to be a slight bias - at least a slight one - in favour of regulating ontological discourse in such a way as to reserve the word "part" for designating only part-relations between homocategorial entities, while the word "constituent" is to serve as the more general mereological term (such that every part is a constituent, but not vice versa).

In any case, there is not only a heterocategorial part-relation between universals and states of affairs, but also a homocategorial part-relation between universals of the same type. Consider the simplest case: generally defined properties of individuals, that is, monadic (predicative) universals that compose a state of affairs with each individual, but with no non-individual. Let $\mathrm{P}$ and $\mathrm{P}^{\prime}$ be two such properties; then $\mathrm{P}$ is an intensional part of $\mathrm{P}^{\prime}$ if, and only if, for all individuals $\mathrm{X}$, (the state of affairs) $[\mathrm{P}, \mathrm{X}]$ is an intensional part of (the state of affairs) $\left[\mathrm{P}^{\prime}, \mathrm{X}\right]$. According to this, the property of being extended is an intensional part of the property of being coloured. Or consider a slightly more complex case: generally defined dyadic relations between individuals, that is, dyadic (predictative) universals that compose a state of affairs with each ordered pair of individuals, but with no ordered pair that has a non-individual as one of its component. Let $\mathrm{R}$ and $\mathrm{R}^{\prime}$ be two such relations; then $\mathrm{R}$ is an intensional part of $\mathrm{R}^{\prime}$ if, and only if, for all individuals $\mathrm{X}$ and $\mathrm{Y}$, (the state of affairs) $[\mathrm{R}, \mathrm{X}, \mathrm{Y}]$ is an intensional part of (the state of affairs) $\left[\mathrm{R}^{\prime}, \mathrm{X}\right.$, 
Y]. According to this, the relation Living-earlier-than is an intensional part of the relation Being-a-progenitor-of.

The above examples are instances of a general principle, stating the general reducibility of intensional parthood for universals to intensional parthood for states of affairs: Universal $U$ is an intensional part of universal $U^{\prime}$ if, and only if, $U$ and $U^{\prime}$ compose states of affairs with the very same sequences of entities and, for every sequence $Q$ of entities with which $U$ composes a state of affairs, it is true that the state of affairs $U$ composes with $Q$ is an intensional part of the state of affairs that $\mathrm{U}^{\prime}$ composes with Q. Clearly, according to this, the principles of the intensional mereology of universals will be consequences of the principles of the intensional mereology of states of affairs.

\section{References}

Armstrong, D. M. (1978). Universals and Scientific Realism, 2 vols., Cambridge: Cambridge University Press.

Armstrong, D. M. (1997). A World of States of Affairs, Cambridge: Cambridge University Press.

Künne, W. (1982). "Criteria of Abstractness", in B. Smith (ed.), Parts and Moments. Studies in Logic and Formal Ontology, Munich: Philosophia, 401-437.

Lewis, D. (1986). On the Plurality of Worlds, Oxford: Blackwell.

Meixner, U. (1997). Axiomatic Formal Ontology, Dordrecht: Kluwer.

Meixner, U. (2006). The Theory of Ontic Modalities, Heusenstamm: ontos.

Simons, P. (1987). Parts. A Study in Ontology, Oxford: Clarendon Press.

Ridder, L. (2002). Mereologie, Frankfurt a. M.: Klostermann. 